\title{
Mental Fatigue Evaluation of Surgical Teams during a Regular Workday in a High-Volume Tertiary Healthcare Center
}

\author{
Bogdan Petrut $^{a} \quad$ loana Berindan-Neagoe ${ }^{a}$ Dragos I. Feflea $^{b}$ \\ Maximilian Hogea ${ }^{c}$ Sergiu Pasca $^{d}$ Cristina E. Bujoreanu ${ }^{b}$ Thomas Bschleipfer $^{\mathrm{e}}$ \\ a Iuliu Hatieganu University of Medicine and Pharmacy, Cluj-Napoca, Romania; ${ }^{b}$ Department of Urology, \\ Prof. Dr. Ion Chiricuta Institute of Oncology, Cluj-Napoca, Romania; 'The Regional Institute of Oncology, lasi, \\ Romania; ${ }^{d}$ Department of Functional Genomics, Prof. Dr. Ion Chiricuta Institute of Oncology, Cluj-Napoca, Romania; \\ e Clinic for Urology, Andrology and Pediatric Urology, Clinics of Nordoberpfalz AG, Weiden, Germany
}

\section{Keywords}

Mental fatigue $\cdot$ Cognitive performance $\cdot$ Surgeons .

Urologic oncology

\begin{abstract}
Introduction: Considering the complex set of manual and psychological tasks a surgeon has to perform during the day, it is very important to assess the surgeon's fatigability, reaction time, attention, and memory. Objective: Here, we wanted to determine the mental fatigue status of surgeons and how their abilities are affected through a regular workday. Methods: We included 3 senior urologists and 6 urology residents. In a set period of time, we assessed their fatigue through self-assessed fatigue, Samn-Perelli score, and Karolinska sleepiness scale score. Further, reaction time, attention, and memory correlated with the number of the operations in the day, and total number of operations in that day were assessed. Results: As the number of tasks increases, and as the surgeons advance to the end of the workday, they become more fatigued, reaction time ultimately increases, and
\end{abstract}

attention and memory become slightly altered. Conclusions: Complications resulting from the fatigue of surgeons could be serious. Their performance status and skills decrease as they perform more tasks or advance through the day.

(c) 2020 S. Karger AG, Basel

\section{Introduction}

Mental fatigue is a psychobiological state caused by prolonged periods of demanding cognitive activity [1], ubiquitous in clinical practice and not only a problem of our times. The combination of long working hours, difficult mental and physical tasks, and requirement for constant reliable results put a strain on every member of the medical staff, but even sooner on the people responsible for the correct constant care of patients. However, the

Bogdan Petrut and loana Berindan-Neagoe contributed equally to this study.
Cristina E. Bujoreanu, MD

Department of Urology, Prof. Dr. Ion Chiricuta Institute of Oncology E. Grigorescu Street 89 RO-400305 Cluj-Napoca (Romania)

E-Mail bujoreanucristina@yahoo.com 
concept is difficult to define, and there is no standardized way to evaluate fatigue in an objective manner. Although the effects of mental fatigue on the skilled performance of other high responsibility careers (such as vehicle drivers and air pilots [2]) have been evaluated, there are little data on how this parameter affects surgeons, and even less on the results of surgery. Surgical procedures require a complex set of manual and psychological tasks as well as eyehand coordination, which in turn entails a prolonged period of demanding cognitive activity in which attention, memory, and reaction time play a key part. Minimally invasive techniques are even more demanding due to the constraints of the restrained access and lack of manual feedback. Surgeons are required to maintain full focus during operations in order to avoid complications, but this may become more difficult in the presence of fatigue $[3,4]$. The most important study that addressed this issue was performed by McCormick's group. It evaluated the incidence and severity of orthopedic surgical resident's fatigue and the predicted risk of medical error [5].

The objective of this study is to evaluate the mental fatigue of surgeons in a clinical setting using several fatigue scales and cognitive tests and attempt to discover any links these measurements may have with clinical outcomes.

\section{Materials and Methods}

\section{Study Design}

In our study, we included 3 senior urologists and 6 urology residents, all men, performing operations in an academic tertiary cancer center in Cluj-Napoca, Romania. We evaluated the subjects included in our study between November 2014 and January 2015. During this time period, senior urologists performed 4 operations/ day and the urology residents assisted senior urologists in the operations. Also, during the recorded interval, the senior doctors attended the hospital outpatient department on 3 operative days. In the abovementioned days, surgeons performed 3 operations/OR day. The surgeries that were considered more difficult were performed earlier during the day, usually on first position, and less difficult surgeries were performed later in the day. The reasons would be the availability of more resources at the start of the day such as blood transfusion stock or not intending to keep biologically fragile patients dehydrated or fasting for many hours. All surgeries were performed for oncologic pathologies.

\section{Evaluation Methods}

In our study population that was composed of men only, as there were no women working as surgeons in our center, we evaluated fatigue, attention, memory, and reaction time. We made the evaluation before and after each surgery during 15 different operating days under normal equivalent workload. To assess the fatigue of the surgeons, we used the Karolinska sleepiness scale [6], the Samn-Perelli 7-point scale and a self-assessed numerical fatigue rating scale $15 \mathrm{~min}$ prior and after each procedure. To assess attention, memory, and reaction time, we used a computer-based battery of psychological tests $15 \mathrm{~min}$ prior and after each procedure (Inquisit Lab Academic software). Reaction time was evaluated by measuring the response time to displayed visual stimuli; memory was evaluated using pattern recall exercise (an animation showed numbers being connected in a pattern which the subjects had to recall from memory and redraw). Attention involved placing various displayed symbols into predefined categories (colors, shapes, numbers). The same computer was used for the testing that was made in a separate room next to the operating room, with no distractions. All participants were in good physical shape with no impairments that could jeopardize the results. We also recorded several perioperative parameters related to the surgery or to the surgeon.

\section{Data Analysis}

We performed statistical analysis of data using the MannWhitney $U$ test and using a significance level of $p<0.01$ for all analyses. We compared presurgery with postsurgery data: data between the 1st, $2 \mathrm{nd}, 3 \mathrm{rd}$, and 4 th operations and data between regular OR days and days in which the senior doctors also performed consultations in the outpatient department.

\section{Results}

Preoperative fatigue increased significantly during the 1st, 2nd, 3rd, and 4th operations, as reflected by the different fatigue scales: Karolinska sleepiness scale, SamnPerelli fatigue scale, and self-assessed fatigue scale. There was an increase in fatigue scores by $98.44 \%$ (self-assessed scored), 67.95\% (Samn-Perelli scale), 88.93\% (Karolinska sleepiness scale) from the start of the first operation to the start of the 4th operation of the day. There was a significant decrease in attention and memory score when comparing the scores from the 1st versus the 2nd operation, 2nd and 3rd, and 3rd and 4th. Preoperative reaction time also increased significantly between the operations. Preoperative data are presented in Figure 1.

Postoperative attention and memory scores as well as reaction time increased significantly after the 1 st and 2 nd surgeries, while fatigue scores increased (Fig. 2). Postoperative attention and memory scores recorded in the first two surgeries were better than those recorded after the same surgeries. Attention score was improved by $13.17 \%$ $(p<0.001)$ after the 1 st surgery, with an increase of $9.73 \%$ $(p<0.001)$ after the $2 \mathrm{nd}$. Similarly, the memory score was improved by 19.77 and $19.27 \%$, respectively $(p<0.001)$. Postoperative reaction time improved significantly when compared to the preoperative reaction time of the first two surgeries of the day. There was also a drop of 7.97 and $3.40 \%$, respectively $(p<0.001)$, in reaction time after the 



Fig. 1. Comparison of preoperative variables before and after each surgery. $p$ values of more than 0.05 are marked with black squares.



Fig. 2. Comparison of postoperative variables. 


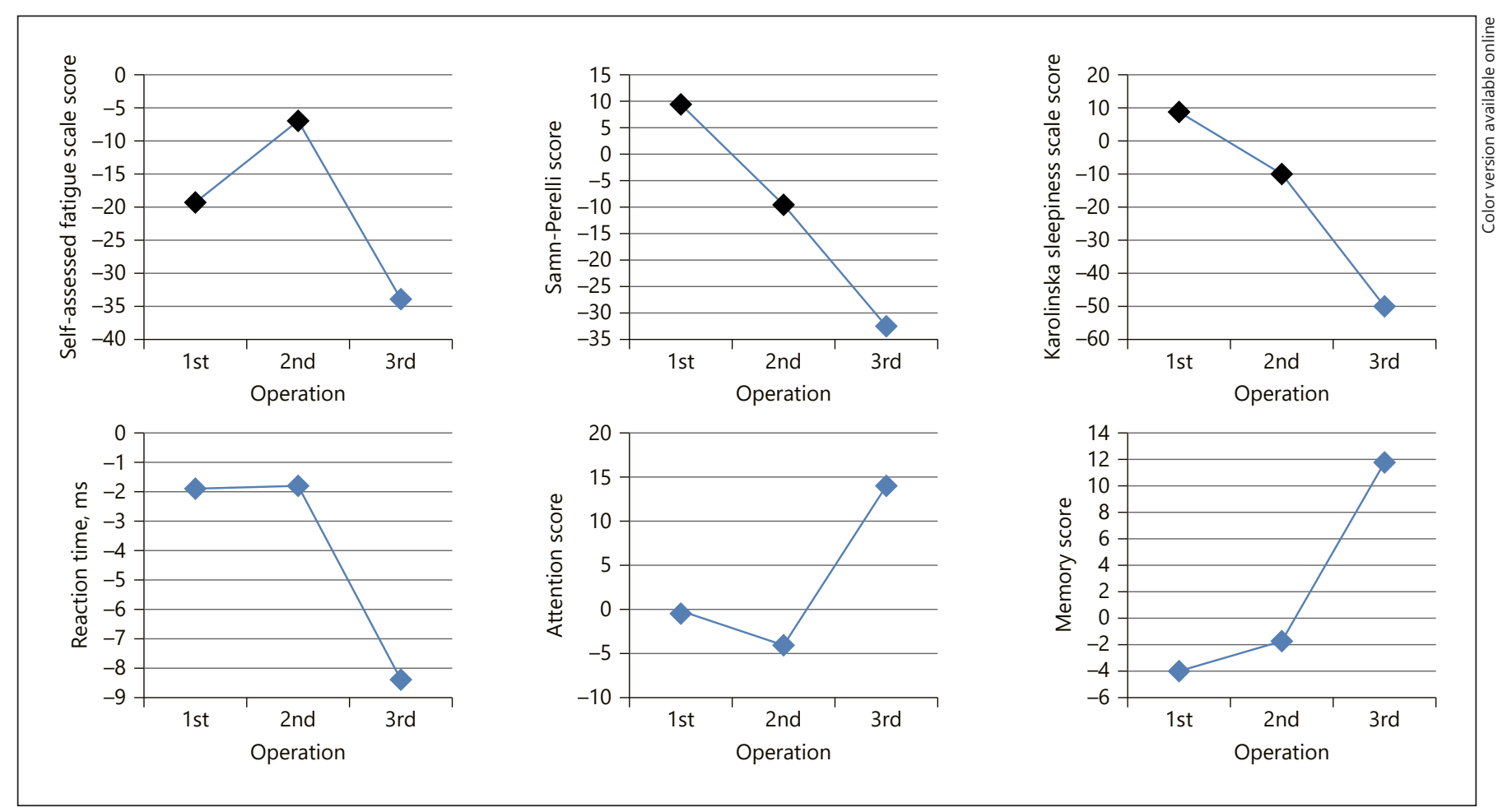

Fig. 3. Comparison of variables on outpatient clinic days. $p$ values of more than 0.05 are marked with black squares.

1st and 2nd surgery when the results were compared with those recorded before the start of the mentioned surgeries, but the scores gradually deteriorated afterwards. Attention scores recorded after the 1st operation dropped by $34.38 \%(p<0.001)$ when compared to those recorded after the 4th operation; similarly, memory scores dropped by $41.07 \%(p<0.001)$, and reaction time increased by $34.69 \%(p<0.001)$. After the 3rd and 4th surgeries, attention and memory scores decreased significantly, while reaction time increased significantly. Data collected from the fatigue scales showed that there was a significant increase in score after each operation.

Worst scores both for cognitive test and fatigue scale were attained if the subject performed consultations in the outpatient clinic between surgeries. There was also a significant drop in scores in attention and memory, as well as in fatigue scales, after the surgeons performed consultations in the outpatient clinic when compared to regular operative days (Fig. 3).

When comparing the change in score modification from the 2 nd and 3rd operations and 3rd and 4th, we noticed that the onset of fatigue reflected by the fatigue scales and cognitive tests was linear. There was a significant change in score modification regarding attention, memory, reaction time, and Karolinska sleepiness scale score between the first two operations of a regular OR day (Fig. 4).

At the present moment, we take into account these results, and we place challenging and complicated surgeries in the second position on the operatory schedule.

\section{Discussion}

Mental fatigue is ubiquitous in clinical practice, difficult to define, and even more difficult to evaluate in an objective manner. Although the effects of mental fatigue on the skills of both drivers [7-9], air pilots [10-13], and financial workers [14] have been evaluated, data on mental fatigue and its implications in a clinical context are scarce in the medical literature. Although aviation and surgery are different domains, they show similarities, and there are studies that prove that there are concepts used by air pilots that can be translated to surgery [15]. Also, there have been studies comparing mental fatigue between doctors and firefighters [16]. The level of cognitive performance in a highly stressful job with life and death situations is particularly important, and it may be affected 


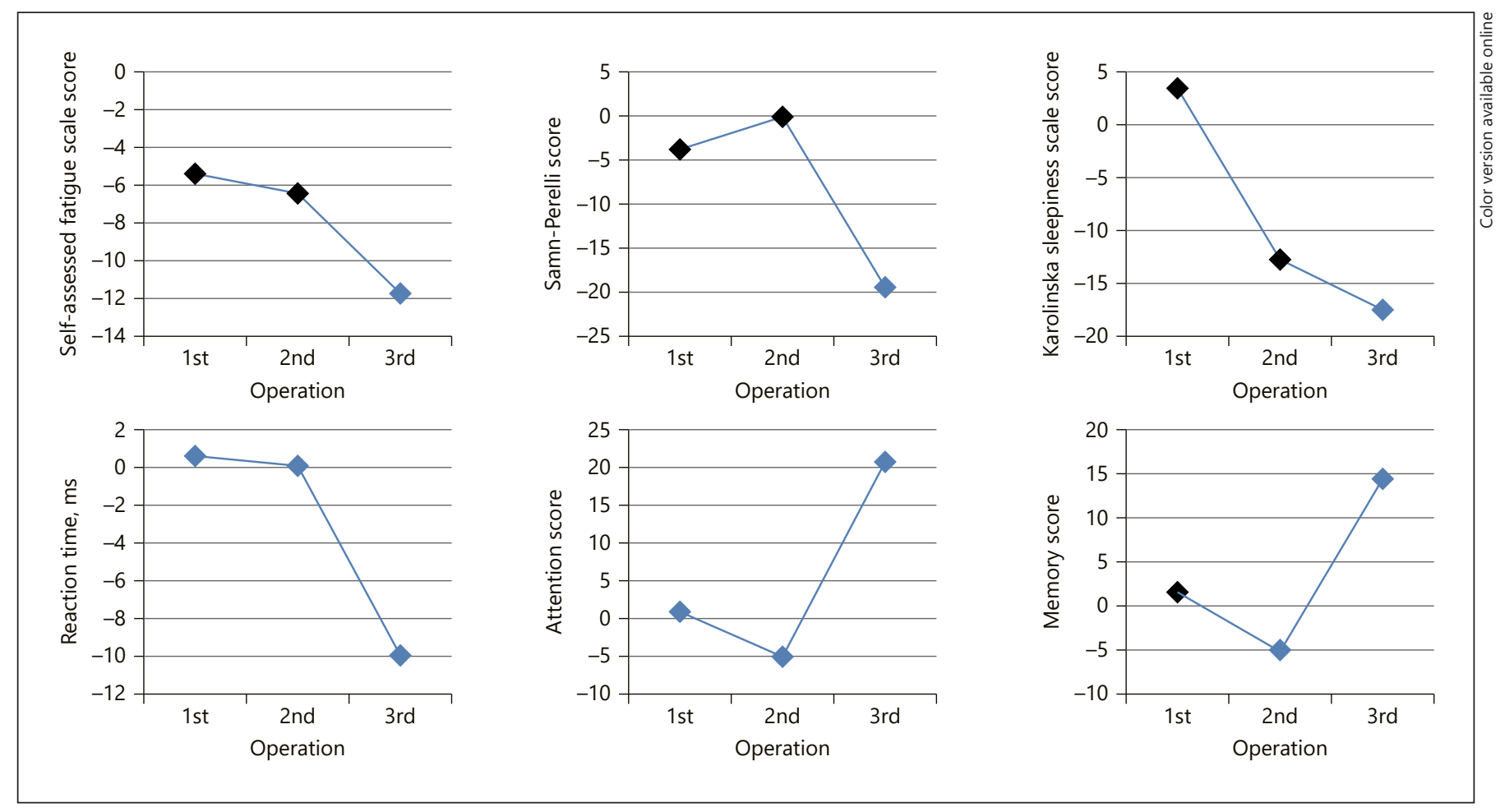

Fig. 4. Comparison of change in score between operations on a regular OR day. $p$ values of more than 0.05 are marked with black squares.

by mental fatigue. Surgeons face challenging situations also during operations where the quality of the surgical act cannot be compromised even in stressful situations. Knowing more about our performance as surgeons can help us improve the management of our skills taking into account the type of tasks we have to perform.

Recently, there has been interest in evaluating mental fatigue of doctors in training, but those studies focused more on sleep deprivation-related fatigue [17-19]. Surgical procedures, especially laparoscopic, require a complex set of manual tasks as well as eye-hand coordination which in turn entail a prolonged period of demanding cognitive activity in which attention, memory, and reaction time play a key part. Still, surgeons are required to maintain full focus during operations in order to avoid fatigue-related complications.

Generally, the most demanding surgeries from a technical point of view were the most time consuming. Such operations were usually performed at the start of the operative day, when the surgeons were supposedly more rested. This was confirmed by our data, the subjects achieving the best scores both for sleepiness and cognitive function items as well as reaction time before and after the first surgery of the day when compared to data re- corded before the 2 nd, 3 rd or 4 th operation. Somewhat surprisingly, our data showed that although the surgeons and the residents were feeling subjectively more tired after the 1st and 2nd surgeries, as reflected by the fatigue scales scores, attention and memory items, as well as reaction time were significantly better than those recorded before the operation. This might be due to an increased level of concentration during surgery, but was no longer the case with subsequent operations when the scores gradually deteriorated in comparison to those recorded before the same operations.

When comparing the preoperative data recorded before the 1st, 2nd, 3rd, and 4th surgeries, there was, as expected, a gradual increase in sleepiness scale scores and reaction time as well as a decrease in memory and attention scores. The results were similar when the postoperative data were compared. By the end of the last surgery, the subjects were feeling rather sleepy and moderately tired (according to the Karolinska sleepiness scale and Samn-Perelli 7-point scale). This difference between preand post-task fatigue has been demonstrated in other studies [20,21]. In contrast, there are studies that show that fatigue does not significantly affect surgeons' performance [22]. 
The biggest differences in attention score and reaction time were those between the 1st and 2nd and 3rd and 4 th surgeries, while changes were similar between the 2 nd and 3rd surgeries. Also, there was a significant change in the drop on memory scores between the 1st and 2 nd operation. The increase in attention and memory was significantly higher during the 1st surgery than during the 2 nd surgery. Also, the drop in attention and memory during the 3 rd surgery was significantly lower compared with the 2 nd. This modification in skills occurring after performing a task or a set of tasks can be seen in other specialties, like trauma surgery, where a decrease in trauma residents' motor skills was observed after a call [23].

The onset of fatigue as reflected by reaction time, attention, and memory scores was gradual. When comparing the change in fatigue scale scores between the start and the end of the operations, we could see that there were no significant differences between the 1 st and 2 nd, the 2nd and $3 \mathrm{rd}$, and finally the $3 \mathrm{rd}$ and 4 th operations. When comparing the changes in attention and memory scores, there was a significant change in score drop between the 1 st and 2 nd operations. As mentioned above, difficult operations were performed early, and less difficult ones were performed later in the day. Still, the drop in scores was similar throughout the day, meaning that in fact, even less difficult surgeries, done later in the day, were taking their toll as much as difficult operations.

Clinical activity, especially performing numerous consultations in a busy outpatient setting can be very tiring, and demanding a considerable amount of mental resources. Our results showed that when the subject had both operations and other clinical activities on the same day, their fatigue and attention scores as well as reaction time were significantly worse when compared to days with a similar operative schedule but without outpatient consultations.

The senior urologist performed endourological, open, and laparoscopic procedures, each requiring a different set of psychomotor skills which in turn involve different levels of fatigue. It would have been interesting to compare the effects of these different types of procedures on surgeons' mental fatigue, but due to the limited number of surgeries involved in this study no conclusion could be drawn. Of course, a larger number of entries would have been preferable, but taking the tests before and after each procedure is very time-consuming.

The time spent relaxing (or not) between operations could have an influence over the preoperative scores, but again, the manner in which this time was spent by the surgeon is hard to quantify. Also, the time spent between operations is proportional to the preparations needed for the following surgery and might not reflect the time needed by the surgeon to rest in order to have better scores. This was the reason why we chose not to incorporate this variable in the measurements.

In a study by McCormick et al. [5], the subjects were feeling tired in $48 \%$ of their awake time mainly because of sleep deprivation due to frequent night calls. This was not case here, as this study involved only surgeons who were not on night calls the previous night.

Attention, memory and reaction time were tested in an "artificial" environment that differs substantially from the intraoperative setting in which other factors may interfere. Therefore, it can be speculated that in a real setting (surgery) these cognitive functions might be augmented due to the stress level involved by such settings. Because the subjects were able to view the scores and compared them to their colleagues after completing the tests, there was a certain degree of rivalry which led to a higher degree of focus in performing them, narrowing the bias-generating gap between real and experimental setting.

Several factors could influence mental fatigue, such as environmental ones, number of hours the surgeon slept the night before, or if the surgeon was on call in the present week and had recent sleepless nights, the time of starting the first surgery, if the surgeon has health problems such as vertebral column disorders, venous insufficiency, the presence of menstruation for female surgeons. One serious problem that can appear is burnout, which can diminish a surgeon's skills even more in a normal day [24]. Considering the great importance of this fact, there are studies that are analyzing methods through which surgeons can get past fatigue, for example by using caffeine or modafinil $[25,26]$. Also, the effects of mental fatigue on surgeons are approached more often than ever [27].

The results of this study need to be verified by further studies, perhaps involving a greater number of subjects. This could prove to be a very difficult task because taking these tests can be time consuming in an already busy surgeon's schedule.

Knowing more about measuring mental fatigue may help surgeons better manage the type of task to be performed at a given time. These results could help organize an optimal schedule for the planned operations, by taking into account the degree of difficulty of the surgeries and factors that could influence the performance of the surgeon. 
Currently, we are investigating whether associating the perceived or objective mental fatigue and performance status of the surgeon with the degree of the difficulty of a surgery when organizing the surgical schedule in a working day would affect work efficiency along with factors that could influence it. This could imply a way of improving the medical care offered to our patients, because knowing more about our performance as surgeons can help us improve the management of our skills taking into account the type of tasks we have to perform.

\section{Conclusions}

Complications resulting from surgeons' fatigue could have potentially serious outcomes for patients, and this aspect is rarely taken into account by medical policy makers. Attributing complications to mental fatigue could be particularly difficult since mental fatigue is influenced by many internal and external factors that are hard to quantify. Also, there are multiple factors that lead to complications following surgery, and it would be difficult to identify those complications that were fatigue-related.

Mental fatigue is ubiquitous in clinical practice, and its effects could increase medical error rate. This study offers an insight into how a day of work in a high-volume tertiary center affects surgeons' memory, attention, and reaction time as well as their level of fatigue. These results can help organize an optimal surgical schedule for the operatory week, taking into account the degree of diffi- culty of the surgeries and mental fatigue-related efficiency. Further studies should be performed to study the factors that can influence the performance of the surgeons.

\section{Statement of Ethics}

Proper consideration has been given to any possible ethical issues, and informed consent was obtained from all individual participants involved in the study. However, an approval from an ethics commission was not applicable as this is not a clinical trial.

\section{Disclosure Statement}

All involved authors have no conflicts of interest to declare.

\section{Funding Sources}

This research did not receive any specific grant from funding agencies in the public, commercial, or not-for-profit sectors.

\section{Author Contributions}

Bogdan Petrut: substantial contributions to the presented work - study design, data acquisition, analysis, and interpretation. Ioana Berindan-Neagoe: revising the work critically for important intellectual content. Dragos Ioan Feflea: data acquisition. Maximilian Hogea: data acquisition. Sergiu Pasca: statistical analysis. Cristina Eliza Bujoreanu: drafting the manuscript. Thomas Bschleipfer: interpretation of results, conception of the study.

\section{References}

1 Marcora SM, Staiano W, Manning V. Mental fatigue impairs physical performance in humans. J Appl Physiol (1985). 2009 Mar; 106(3):857-64.

2 Goode JH. Are pilots at risk of accidents due to fatigue? J Safety Res. 2003;34(3):309-13.

3 Lal SK, Craig A. A critical review of the psychophysiology of driver fatigue. Biol Psychol. $2001 \mathrm{Feb} ; 55(3): 173-94$.

4 Lal SK, Craig A. Driver fatigue: electroencephalography and psychological assessment. Psychophysiology. 2002 May;39(3):313-21.

5 McCormick F, Kadzielski J, Landrigan CP, Evans B, Herndon JH, Rubash HE. Surgeon fatigue: a prospective analysis of the incidence, risk, and intervals of predicted fatiguerelated impairment in residents. Arch Surg. 2012 May; 147(5):430-5.

6 Gillberg M, Kecklund G, Akerstedt T. Relations between performance and subjective ratings of sleepiness during a night awake. Sleep. 1994 Apr;17(3):236-41.
7 Ronen A, Oron-Gilad T, Gershon P. The combination of short rest and energy drink consumption as fatigue countermeasures during a prolonged drive of professional truck drivers. J Safety Res. 2014 Jun;49:3943.

8 Zhang X, Zhao X, Du H, Rong J. A study on the effects of fatigue driving and drunk driving on drivers' physical characteristics. Traffic Inj Prev. 2014;15(8):801-8.

9 Lim SM, Chia SE. The prevalence of fatigue and associated health and safety risk factors among taxi drivers in Singapore. Singapore Med J. 2015 Feb;56(2):92-7.

10 Stoner JD. Aircrew fatigue monitoring during sustained flight operations from Souda Bay, Crete, Greece. Aviat Space Environ Med. 1996 Sep;67(9):863-6.

11 Petrie KJ, Powell D, Broadbent E. Fatigue selfmanagement strategies and reported fatigue in international pilots. Ergonomics. 2004 Apr;47(5):461-8.
12 Jackson CA, Earl L. Prevalence of fatigue among commercial pilots. Occup Med (Lond). 2006 Jun;56(4):263-8.

13 Vejvoda M, Elmenhorst EM, Pennig S, Plath G, Maass H, Tritschler K, et al. Significance of time awake for predicting pilots' fatigue on short-haul flights: implications for flight duty time regulations. J Sleep Res. 2014 Oct;23(5): 564-7.

14 Metlaine A, Sauvet F, Gomez-Merino D, Elbaz M, Delafosse JY, Leger D, et al. Association between insomnia symptoms, job strain and burnout syndrome: a cross-sectional survey of 1300 financial workers. BMJ Open. 2017 Jan;7(1):e012816.

15 Aerden D, Smets D, Poelaert J, Oste J, Van den Brande P. Fighting human error: what surgeons can learn from aviators. Acta Chir Belg. 2014 Jul-Aug;114(4):228-32. 
16 Sun B, Hu M, Yu S, Jiang Y, Lou B. Validation of the Compassion Fatigue Short Scale among Chinese medical workers and firefighters: a cross-sectional study. BMJ Open. 2016 Jun; 6(6):e011279.

17 Mansukhani MP, Kolla BP, Surani S, Varon J, Ramar K. Sleep deprivation in resident physicians, work hour limitations, and related outcomes: a systematic review of the literature. Postgrad Med. 2012 Jul;124(4):241-9.

18 Amirian I. The impact of sleep deprivation on surgeons' performance during night shifts. Dan Med J. 2014 Sep;61(9):B4912.

19 Saadat H, Bissonnette B, Tumin D, Thung A, Rice J, Barry N, et al. Time to talk about workhour impact on anesthesiologists: the effects of sleep deprivation on Profile of Mood States and cognitive tasks. Paediatr Anaesth. 2016 Jan;26(1):66-71.
20 Di Stasi LL, McCamy MB, Macknik SL, Mankin JA, Hooft N, Catena A, et al. Saccadic eye movement metrics reflect surgical residents' fatigue. Ann Surg. 2014 Apr;259(4): 824-9.

21 González-Sánchez M, González-Poveda I, Mera-Velasco S, Cuesta-Vargas AI. Comparison of fatigue accumulated during and after prolonged robotic and laparoscopic surgical methods: a cross-sectional study. Surg Endosc. 2017 Mar;31(3):1119-35.

22 Olasky J, Chellali A, Sankaranarayanan G, Zhang L, Miller A, De S, et al. Effects of sleep hours and fatigue on performance in laparoscopic surgery simulators. Surg Endosc. 2014 Sep;28(9):2564-8.

23 Gerdes J, Kahol K, Smith M, Leyba MJ, Ferrara JJ. Jack Barney award: the effect of fatigue on cognitive and psychomotor skills of trauma residents and attending surgeons. Am J Surg. 2008 Dec;196(6):813-9; discussion 819-20.
24 Bertges Yost W, Eshelman A, Raoufi M, Abouljoud MS. A national study of burnout among American transplant surgeons. Transplant Proc. 2005 Mar;37(2):1399-401.

25 Sugden C, Housden CR, Aggarwal R, Sahakian BJ, Darzi A. Effect of pharmacological enhancement on the cognitive and clinical psychomotor performance of sleep-deprived doctors: a randomized controlled trial. Ann Surg. 2012 Feb;255(2):222-7.

26 Franke AG, Bagusat C, McFarlane C, Tassone-Steiger T, Kneist W, Lieb K. The Use of Caffeinated Substances by Surgeons for Cognitive Enhancement. Ann Surg. 2015 Jun; 261(6):1091-5.

27 Campbell DA Jr. Physician wellness and patient safety. Ann Surg. 2010 Jun;251(6):1001-2. 